\title{
Microfluidic formation of highly monodispersed multiple cored droplets using needle-based system in parallel mode
}

\author{
Zheng Lian ${ }^{1}$ 2§, Yue Chan ${ }^{3 \S}$, Yang Luo², Xiaogang Yang ${ }^{2}$, Kai Seng Koh ${ }^{4}$, Jing Wang ${ }^{5}$, George Zheng \\ Chen $^{6,7}$, Yong Ren ${ }^{2,8^{*}}$, and Jun $\mathrm{He}^{6 *}$ \\ ${ }^{1}$ International Doctoral Innovation Centre, University of Nottingham Ningbo China, Ningbo, China \\ 2Department of Mechanical, Materials and Manufacturing Engineering, University of Nottingham Ningbo China, Ningbo, China \\ IInstitute for Advanced Study, Shenzhen University, Shenzhen, China \\ ${ }^{4}$ School of Engineering and Physical Sciences, Heriot-Watt University Malaysia, Putrajaya Malaysia \\ ${ }^{5}$ Department of Electrical and Electronic Engineering, University of Nottingham Ningbo China, Ningbo, China \\ ${ }^{6}$ Department of Chemical and Environmental Engineering, University of Nottingham Ningbo China, Ningbo, China \\ ${ }^{7}$ Department of Chemical and Environmental Engineering, University of Nottingham, Nottingham, UK \\ ${ }^{8}$ Research Group for Fluids and Thermal Engineering, University of Nottingham Ningbo China, Ningbo, China \\ $\S$ The authors contribute equally to the reported work. \\ *Email: yong.ren@nottingham.edu.cn; jun.he@nottingham.edu.cn
}

Keywords: microfluidics, multiple cores, double emulsion, off-the-shelf system

\begin{abstract}
Scale-up in droplet microfluidics achieved by increasing the number of devices run in parallel or increasing the droplet makers in the same device can compromise the narrow droplet-size distribution, or requires high fabrication cost, when glass or polymer based microdevices are used. This paper reports a novel way using parallelization of needle-based microfluidic systems to form highly monodispersed droplets with enhanced production rates yet in cost effective way, even when forming higher order emulsions with complex inner structure. Parallelization of multiple needle-based devices could be realized by applying commercially available two-way connecters (TWC) and 3D printed four-way connectors (FWC). The production rates of droplets could be enhanced around fourfold (over 660 droplets/min) to eightfold (over 1300 droplets/min) by TWCs and FWCs, respectively, for the production of the same kind of droplets than a single droplet maker (160 droplets/min). Additionally, parallelization of four-needle sets with each needle specification ranging from $34 \mathrm{G}$ to $20 \mathrm{G}$ allows for simultaneous generation of four groups of polydimethylsiloxane (PDMS) microdroplets with each group having distinct size yet high monodispersity (coefficient of variation (CV) $<3 \%$ ). Up to six cores can be encapsulated in double emulsion using two parallelly connected devices via tuning the Capillary number of middle phase in a range of $1.31 \times 10^{-4}$ to $4.64 \times 10^{-4}$. This study leads to enhanced production yields of droplets and enables formation of groups of droplets simultaneously to meet extensive needs of biomedical and environmental applications, such as microcapsules with variable dosages for drug delivery or drug screening; or microcapsules with wide range of absorbent loadings for water treatment.
\end{abstract}




\section{Introduction}

Microfluidics is characterized by the properties such as minute amount of consumed materials, fast analytical and responding performances, and integration of multiphase functions in the same system. Droplets are the most exploited fluid structures in microfluidics, acting as mixers and more importantly as a template to fabricate functional materials carrying a substance of interest. Droplet microfluidics has been developed rapidly in recent years, as it enables precise control over droplet formation, encapsulations and release kinetics using nonlinear channel geometries, such as T-junctions, co-flowing and flow focusing constrictions, to induce the pinch-off of droplets from a mother stream [1-5], leading to a number of industrial and biomedical applications including food industries [6], cosmetics [7], biological applications [8], pharmaceutics [9], reagent mixing and chemical reactions [10, 11]. Wider applications of droplet microfluidics to industry scale is challenged by the demands to scale up the emulsion generation via either integrating more droplet making units in the same microdevice or increasing the number of devices run in parallel to the desired production volumes, therefore careful considerations have to be made in terms of fabrication cost, material selection, microsystem design and operation reliability [12].

Glass is one of the most extensively used materials to fabricate microdevices for droplet generation, attributing to its excellent physical and chemical properties such as optical transparency and chemical inertness $[13,14]$. Many attempts have been made to attain high-throughput droplet generation, the most common way is to produce numerous glass-based devices and work in parallel [15, 16]. However, it is expensive for fabricating droplet makers in a glass-based substrate as laser ablation is needed, the cost will be dramatically increased especially when fabricating more droplet makers in the same device with a limited size. It requires time-consuming alignment procedures when glass capillaries are used. Moreover, the complexity in control of collected droplet size is increased when it comes to parallelizing multiple glass capillary devices. For example, single emulsion can be formed in co-flowing, and double emulsion can be formed in co-flowing combined with hydrodynamic focusing way using a glass capillary device, it is needed to polish inner capillary tip to get the required size of nozzle, however this is normally done in a manual way, and increase in polydispersity of droplet size distribution will become inevitable. Additional use of syringe pumps is also not obviated.

Hierarchical approaches such as tree, ladder or annular structures are preferable when scaling up microfluidic emulsification in a parallel manner. Although parallelizing step emulsifications in ladder or annular structures by glass microfluidic devices can improve the droplet formation rate [15-19], the manufacturing still requires expensive rigs and specific expertise to process. The abovementioned limitations severely constrain the applications of glass-based microfluidic devices, especially in regard of expanding the yields of droplets. The predicament of glass devices could be rectified using glass with PDMS [20, 21] or other materials, such as Polymethyl methacrylate (PMMA) [22-24], PDMS [25-35] or PDMS with other materials such as polycarbonate or silicon [36, 37], to fabricate multiple chips or channels to extend the operation feasibility. Among all the materials mentioned, PMMA is cheaper and more robust than glass devices and hundreds of channels could be crafted in a PMMA substrate; however, it is restricted by manufacturing precision for channels as the etching or mechanical machining techniques are commonly used in its fabrication, this may severely impact surface roughness of individual channel, 
the formed droplet size has polydispersity with coefficient of variations (CV) in a range of about 3-6\% [2224]. This is relatively higher than those when other materials are used. For instance, highly monodispersed droplets with CV below 3\% can be generated when PDMS device was used [31].

PDMS microfluidic device can be rapidly replicated from a master mold using soft lithography, and rearranged in a compact system in the form of layers or stacks for enhanced production of droplets. However, they normally have very elaborated designs, examples of which include the tree structures [25, 30,32 ], multilevel module with radial arrays [27], the sinoidal shaped main channel intersected by many small shunt channels [33], and millipede structures [31]. These designs can dramatically raise the production rate of droplets to the liter-scale per day, yet compromising the production accuracy ineluctably. This becomes more pronounced when dealing with tens of hundreds of droplet generators. For instance, fabrication errors might be introduced when manually drilling or punching the interconnecting holes, the limited fabrication precision might also lead to high polydispersity of the droplet size distribution [27, 29, 34]. When multiple channels are used, each channel has to couple with the neighbouring channels for parallelization, the even distribution of flows might be hindered by debris, dust, air bubbles, clogging or aggregates of chemicals and fouling by the inner fluids in the channels. These inevitably reduce the actual numbers of active channels or even cause failures of production [29]. It thus increases the difficulty for troubleshooting the faulted channels or altering channel geometries [31], particularly when the microdevices are irreversibly bonded or assembled. The PDMS devices also require the use of large or extra syringe pumps whereas the precise control of flow rates might be compromised or even sacrificed especially when pumping highly viscous fluids into the scalable PDMS devices because of the substantially increased flow resistance [22], [25]. In addition, PDMS necessitates surface wettability modification to render hydrophilicity. However, the uncross-linked PDMS monomers in the bulk would gradually migrate to its surface as a result of the minimum surface energy of the polymer chain, and thus recover to its original hydrophobic behaviour [38]. Furthermore, organic compatibility inhibits the wider application of PDMS on dealing with swellable organics that could result in deformation or collapse of the PDMS microdevice for droplet generation [39-42].

Off-the-shelf needle-based microfluidic devices were firstly reported by Li et al [43]. This versatile costeffective device is regarded as a promising alternative to producing droplets with good control over the size. It is also proficient to generate core-shell double emulsion microdroplets for multiple applications [43]. The needle-based microfluidic devices have been applied to fabricate pristine and two types of porous PDMS microparticles using oil-in-water single emulsion template for treating synthetic wastewater that contained organic compounds [44]. Nevertheless, the production rate is 130 pieces per minute and still quite low, the development of needle-based droplet microfluidics is hindered by insufficient means for scale-up formation of multiple cored droplets without increasing complexity of devices. One can find more applications from double emulsions encapsulating multiple cores, which can carry multiple drugs targeting different diseases simultaneously. This work demonstrates a robust method to produce highly monodispersed $(\mathrm{CV}<3 \%)$ emulsion drops from a co-flowing system on a needle-based microfluidic platform running in parallel mode. With this approach, we can generate core-shell droplets encapsulating up to 6 cores. In particular, we show that the complexity of the resultant emulsions can be controlled by the Capillary number of middle phase. To the best of our knowledge, we report for the first time in this 
paper a viable and facile method using needle based microfluidic system for scale up formation of droplets with high monodispersity and low cost in a parallel mode. Our device is easy to assemble. Significantly, it can generate droplets with enhanced production rates, controllable droplet size and number of inner cores concurrently.

\section{Materials and experimental setup}

The needle based microfluidic system comprises dispensing needles, polypropylene (PP) cross link, and silicone rubber soft tubes. Single emulsion microdroplets were generated by assembling a PP cross link and soft tube where the dispersed phase (DP) was penetrated into the device through the inlet needle at a pre-set flow rate from a syringe pump, and breakup into droplets by the continuous phase (CP) pumped from the perpendicular directions into the cross link, when the shear stress from $C P$ overcomes the interfacial tension between two phases [45] (see Fig.1A, B and inset). Thanks to the hydrodynamic focusing of co-flowing geometry, the need for surface wettability modification upon the channel wall of single emulsion needle device can be obviated. As marked by a white rectangle in Fig. $1 \mathrm{C}$, the outlet needle was located downstream at a distance (i.e., $3 \mathrm{~mm}$ ) away from the inlet of the continuous phase. The zoomed edges of two needles in the flow junction area were highlighted in red in Fig. 1D, together with a blue arc depicting the ejected droplet. Time-lapse images in Fig. 1D depicts that the inner fluid was dragged and sheared by the outer phase and ultimately broken into droplets when shear force become dominant over the interfacial tension. The time interval of each image was about $75 \mathrm{~ms}$ with an error around $25 \mathrm{~ms}$ in Fig. 1D (a) to (d). The interface of the inner fluid then recoiled soon caused by the capillary pressure after the droplet breakup. The same setup can be extended to form the PDMS microparticles where the mixture of PDMS precursor and curing agent (10:1 wt\%) was used as DP; and deionized (DI) water mixed with $5 \mathrm{wt} \%$ polyvinyl alcohol (PVA) was used as CP. The formed droplets were collected in a beaker via the outlet needle whose needle holder was removed to fit into the cross link.

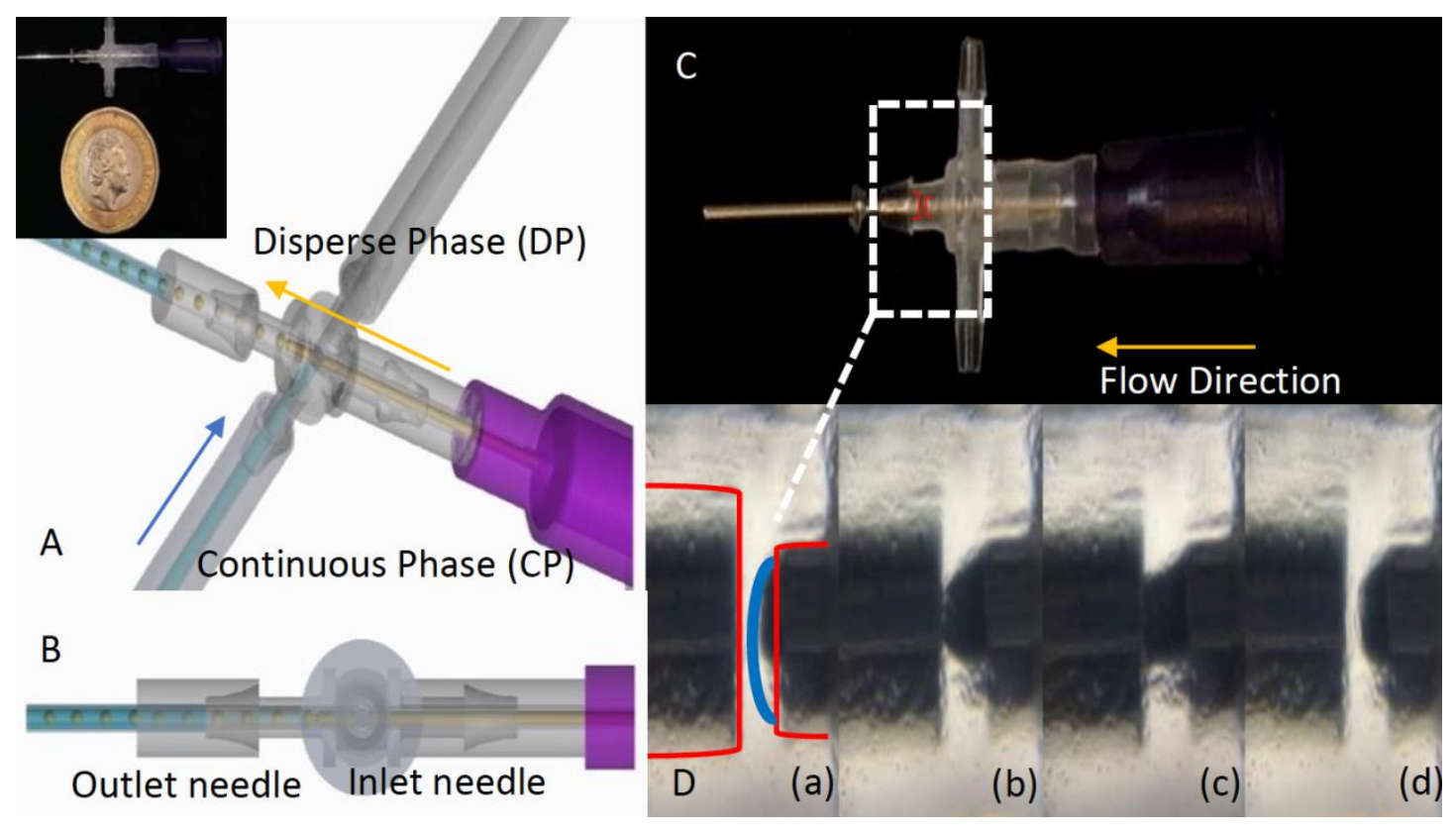

Figure 1. Demonstration of water-in-oil (W/O) single emulsion formation process with needles of 21G20G (21G: I.D. $=510 \mu \mathrm{m}$, O.D. $=800 \mu \mathrm{m}$ and 20G: I.D. $=600 \mu \mathrm{m}$, O.D. $=900 \mu \mathrm{m}$. The device is shown in the 
inset with a coin of one British Pound for scale. (A) Isometric view and (B) Cross section view of the schematic of the needle device for single emulsion microdroplet generation. (C) Photo of the needle device in co-flowing mode and (D) Time-lapse images of the droplet formation in the device, the time interval of each image was about 75 ms with an error around 25 ms as shown in (a) to (d).

Core-shell structured double emulsion microdroplets were fabricated according to Li's design [43]. A needle with length of $50.8 \mathrm{~mm}$, referred to as inner needle, was inserted into the aforementioned single emulsion droplet maker where for clarity, we recall the previous inlet and outlet needles as the middle and outer needles, respectively. The inner phase was introduced through the inner needle, and was then surrounded by the incoming middle phase and together were sheared into double emulsion microdroplets by the outer phase at the junction where middle and outer needles meet. Leakage or clogging occasionally occurred at the T-link section and took place more frequently especially when highly viscous fluids such as PDMS was used. When tuning the flow rate, it might cause high shear stress inside the device and result in fractures or even fluid splashing out of the device. Therefore, it attests to some modifications for the current design to ensure the needle based microfluidic system can function properly, especially at high flow rate conditions. To circumvent the leakage problem and ensure stability of the device, we used a commercially available exquisite three-way link as a replacement of the original T-link section, see Fig. 2A and $B$. The outlet of the link features a snap joint with a screw thread which can firmly be connected with the inner needle and middle needle (see Fig. $2 \mathrm{C}$ ). The design allows rapid replacement of any component if problems occur. The other entrances were securely sealed by glue. The assembled needle device can be applied for formation of double emulsion microdroplets.

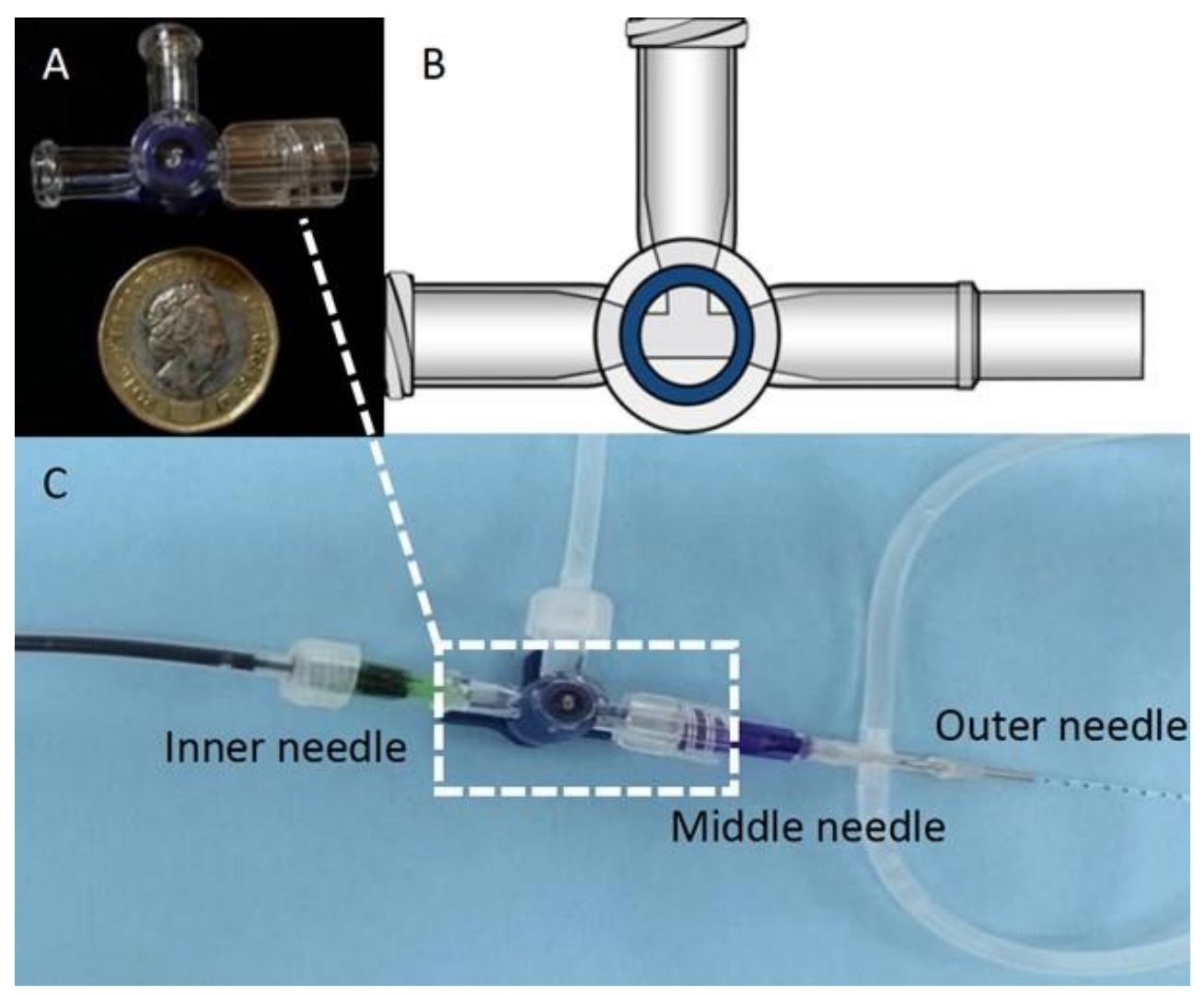

Figure 2. (A) Three-way link and (B) its schematic. (C) Application of three-way link with needle device for production of double emulsion microdroplets. 
The dispensing needles, PP cross link, silicone rubber soft tubes and three-way link were purchased from Taobao. Laboratory syringe pumps (Longer LSP02-1B) from Longer Precision Pump Co., Ltd (Hebei, China) were adopted for delivery of fluids and control of flow rate. PDMS (Sylgard 184 silicone elastomer) were purchased from Dow Corning (USA). PVA and octadecyltrichlorosilane (OTS) were obtained from Sigma Aldrich (Shanghai, China).

PDMS precursors were well mixed and degassed for $20 \mathrm{~min}$ in a desiccator that connected with a water circulation multifunction vacuum pump (SHZ-DIII mode, Henan, China) to remove air bubbles after stirring the PDMS. An optical microscope (N-800F, Ningbo Novel Optics, China) equipped with a camera (QIMAGING, Canada) was employed to observe the formed droplets, the optical images and videos were recorded in a computer. The size of at least 200 droplets were measured by the software Image J for each experiment which has been repeated for 3 times to evaluate the reproducibility of the measurements. CV was calculated as a ratio of the standard deviation (STDV) of droplet sizes and their average diameters to reflect the monodispersity of droplet diameters:

$$
\mathrm{CV}(\%)=\frac{\mathrm{STDV}}{\mathrm{AD}} \times 100 \%
$$

Interfacial tension between PDMS and deionization (DI) water was measured by interface tensiometer (Dataphysics DCAT21, Germany). Viscosities of PDMS were measured by rotational rheometer (Anton Paar Physica MCR-301, Austria).

\section{Design of needle-based system in parallel mode}

\subsection{Two-way connector for double droplet synthesis}

PDMS can be used as a functional material for removing organic substances in water $[45,46]$. The needle devices are capable of producing monodispersed PDMS microdroplets, which can be thermally cured offsite for the organic compound removal [47]. Currently, the handicap is how to massively produce highly monodispersed PDMS microdroplets. The amount of PDMS microdroplets produced by a single needle device is far insufficient to fulfil the demand. To enhance the throughput of PDMS microdroplets, we employed an exquisite and commercially available flow diverter, defined as two-way connectors (TWC), to operate multiple droplet makers simultaneously as demonstrated in Fig. 3 . This connector is as diminutive as $15.8 \mathrm{~mm}$ long with two circle outlets, both of which have the same diameter of $1.5 \mathrm{~mm}$, at each of which the input fluids have an unimpeded access from the syringe to the channels through a soft tube. Fig. 3A shows a snapshot of this unit and Fig. 3B, C and D depict the schematic of this unit, its cross section and bottom view, respectively. It can be equipped at the tip of syringes to evenly apportion the input fluids to all drop makers. As such, two aforementioned droplet makers can be parallelized to generate microdroplets with a narrow size distribution given the same device parameters and flow conditions. This type of connection is demonstrated in Fig. 3E. It is worth mentioning that in our experiments, we actually parallelly connected four sets of the needle devices (21G-20G) to gain an eightfold production rate of PDMS microdroplets in comparison to a single needle device, see the details in Fig. S1 and Supporting Video S1. 


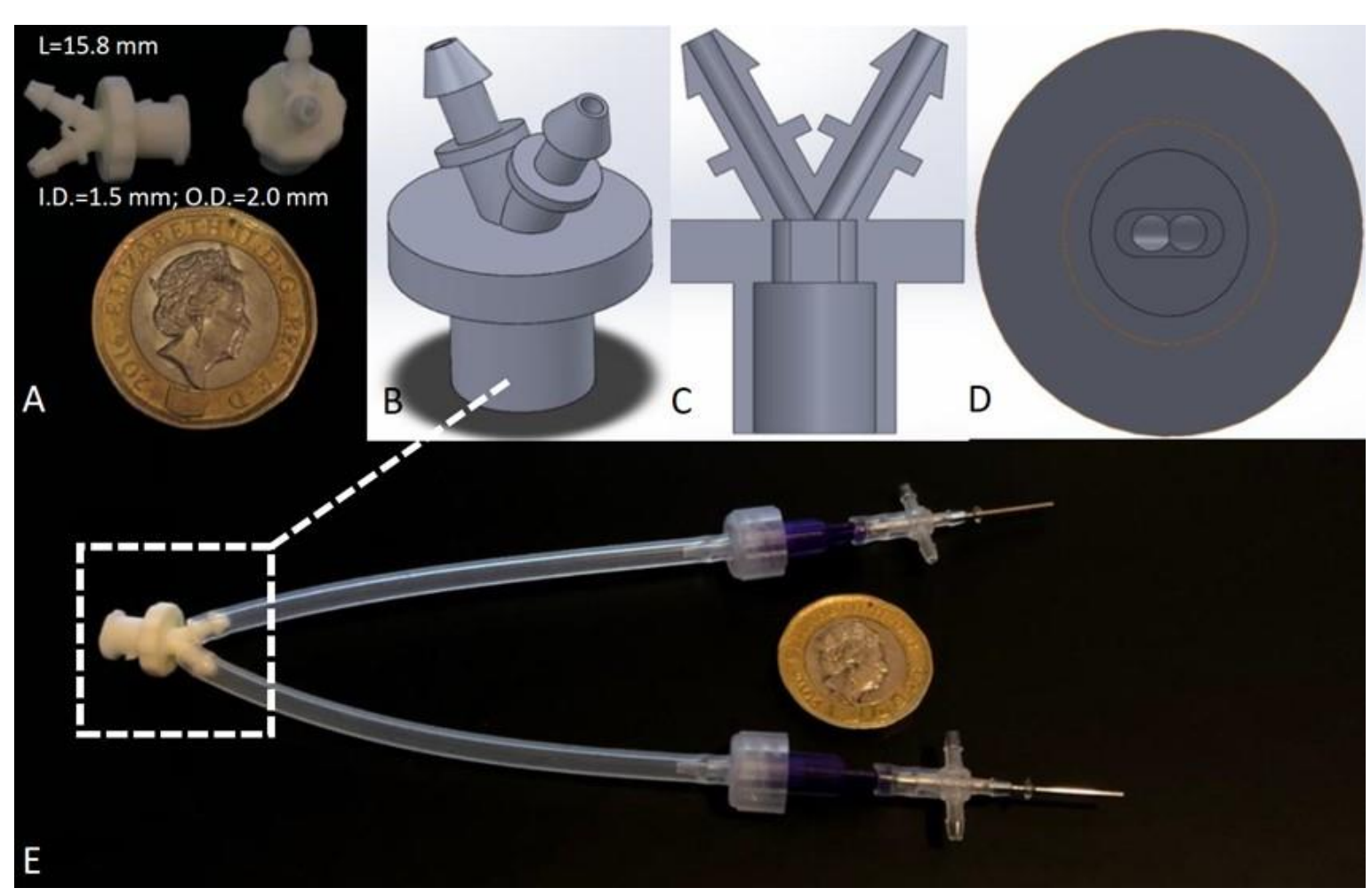

Figure 3. (A) Photo of TWC for parallel connection, (B) its isometric view, (C) cross-section view, (D) bottom view and $(E)$ the application of the TWC for a parallel connection of two single emulsion needle devices. The coin is used for scaling.

\subsection{Four-way connector for quadruple droplet synthesis}

Inspired by the design of Two-way connector, we developed a more powerful flow diverter known as Four-way connector (FWC) using a 3D printing technique. It appends two additional outlets that allow two more channels to be parallelized, as demonstrated in Fig. 4A (from left to right as: side view, top view and an end product). This FWC is $15 \mathrm{~mm}$ long with four equal sized circle (diameter $1.5 \mathrm{~mm}$ ) as its outlets. Fig. $4 B, C, D$ and $E$ display the design drawing, perspective drawing, top view and interior view including the four circle outlets, respectively. The design was made by software SOLIDWORKS and printed by FormlabsForm2 3D printer (USA) with Stereo Lithography Apparatus (SLA) technology using photopolymer resin. The cost for printing one piece of the unit is 0.45 US dollars. With integration of such a 3D printing technique, it is thus possible to achieve four to eight or even more needle devices to be parallelly connected as this unit permits multiple independent connections while saving the use of additional pumps. In Fig. 4F, four needle devices with same needle combination were connected in a parallel mode to form an MFDGs system. We have equipped two such systems containing eight channels to work simultaneously (please refer to Fig. S2 and Supporting Video S2). The droplet production rates were significantly raised up to almost eightfold (over 1300 PDMS drops per minute) than those of a single device (160 droplets per $\mathrm{min}$ ) and a high monodispersity of the droplets was achieved $(\mathrm{CV}<3 \%$ ). 


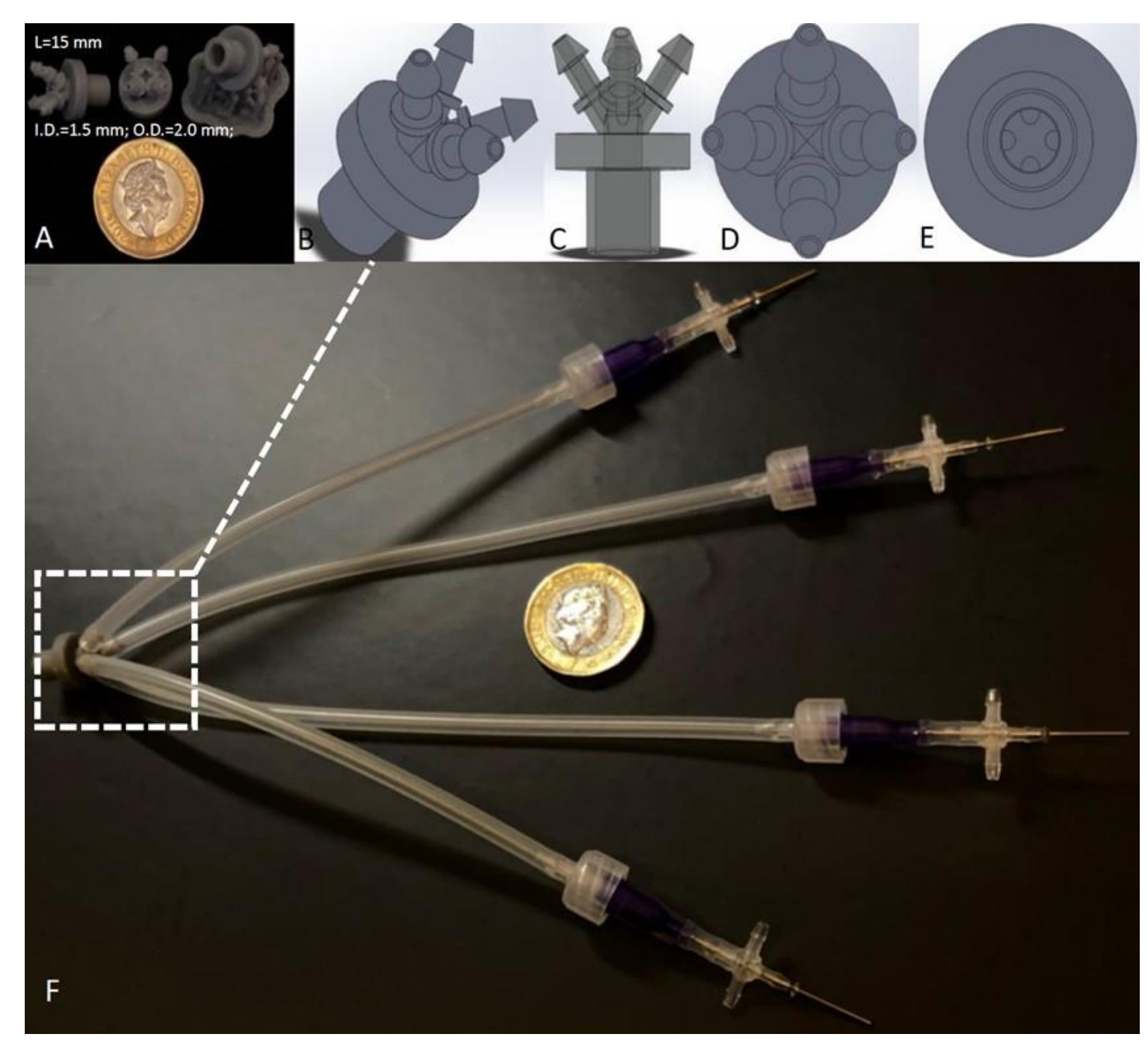

Figure 4. (A) Photo of the 3D printed FWC, (B) isometric view, (C) cross-section view, (D) top view, (E) bottom view, and (F) an FWC-based parallel connection of needle devices for production of PDMS single emulsion microdroplets.

\subsection{Six-way microfluidic component (SWMC)}

On top of the flow diverting units, such as TWC or FWC, used in our experiment, another useful and offthe-shelf component was also employed, which is defined as a six-way microfluidic component (SWMC), as shown in Fig. 5A, B and C. This component connects two sets of needles in a shunt way and the other two inlets for the use of introducing the continuous phase. The inlet needles $(25.4 \mathrm{~mm}$ long) are linked with two soft rubber tubes that can help us not only adjust the needle positions, but also control the distances between inlet and outlet needles. Through use of SWMC, the droplet production rates have been raised up to almost double more than those of a single needle device while more space was saved than the aforementioned connection approaches. A demonstration video of utilization of this unit for production of PDMS microdroplets can be found in Supporting Video S3. In addition, this component is easy to be integrated with TWC or FWC so as to achieve four-channel or even eight-channel parallelization. In Fig. 5D, CP and DP were depicted by the dark blue and yellow arrows, respectively, and the produced droplets were in yellow dots. The center section was marked by red dots in which a distributary of the 
incoming CP (marked by the light blue arrows) filled first before joining a shearing process of CP with the outer split-flow. Similar to this set-up shown in Fig. 5E, two single needle devices can be directly connected in a shunt way by a soft rubber tube, as shown in Fig. 5F and Fig. S3. Table 2 lists the details of droplet productions using TWC, FWC and SWMC, including the production rates, average diameters of droplets and the corresponding $\mathrm{CV}$ values.

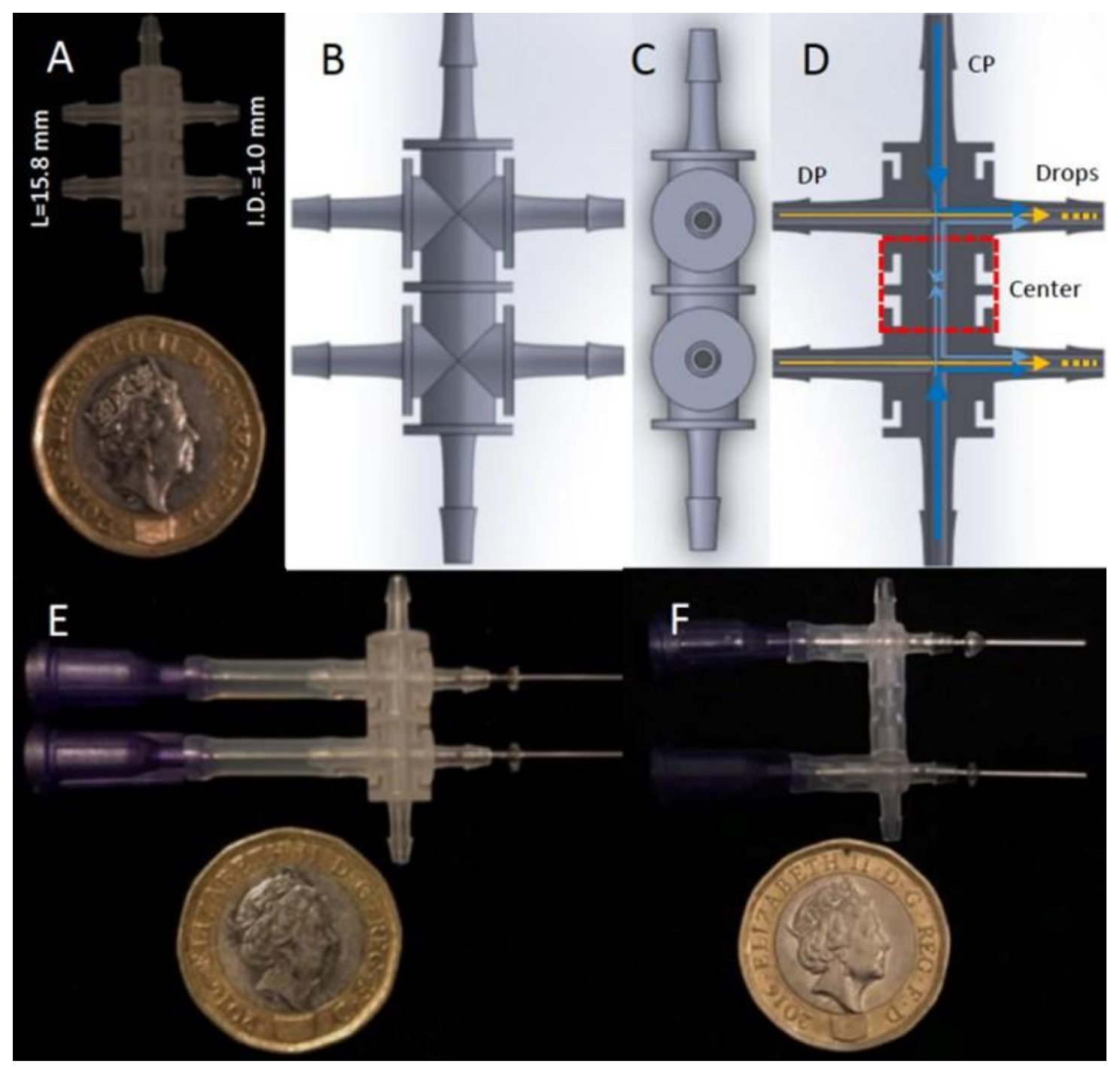

Figure 5. (A) Photo of a SWMC, (B) front view, (C) side view, (D) cross-section view, (E) a shunt connection with needles for production of single emulsions and (F) a shunt connection with two single needle devices. The coin was used for scaling.

\section{Results and Discussion}

\subsection{Single emulsion formation}

The flow rates of dispersed phase $\left(Q_{d}\right)$ and continuous phase $\left(Q_{c}\right)$ were manipulated as 20 and $200 \mu \mathrm{L} / \mathrm{min}$, respectively. Approximately 660 PDMS microdroplets were generated within one minute and an overall CV is as low as $1.9 \%$. The results indicate that the production rates have been raised around triple than that of a single generator (160 pieces per min) while the droplet remained monodispersed. Although a 
paralleled connection inevitably broadens the size distribution due to errors of device fabrication, flow resistance and operations, the droplet formation by this device is highly reproducible since each emulsifier can be controlled independently. Unlike the ladder or tree channel structures, the incoming flow was evenly diverted through the TWC in our experiment, making each droplet maker insensitive to one another. This greatly minimizes interferences among channels and ensures the enhanced throughput of droplets and long-time stability. In addition, the design circumvents the issues that often occurs in PDMS devices such as debris, clogging or fouling that may result in a failure of the entire device [29]. For example, troubleshooting of the bonded PDMS device might dismantle the whole system; the glass device might be easily broken due to the fragility of thin glass capillary. Those issues could be addressed easily by component replacement, giving rise to robustness of the needle-based devices. For instance, needles or cross links can be replaced rapidly if there exist malfunctions. Moreover, the assembling of needle devices is rather user friendly and not labor-intensive. Furthermore, the total costs of one needle devices system in Fig.3C (excluded for the pumps) are just less than 4 US dollars, very cost-effective as compared with glass capillary-based MFDG (detailed costs of units could be found in Table S2).

Endowed by flexible assembling and numerous alternatives of needle combinations, a large variety of needle combinations can be arranged in a parallel manner to produce diversified sizes of droplets simultaneously. In Fig. 6, we parallelly connected four different needle devices with needle sizes of 34G30G, 32G-27G, 25G-21G and 23G-20G, respectively, by TWCs to generate PDMS microdroplets. Given the same flow rate of 20 and $200 \mu \mathrm{L} / \mathrm{min}$ for $Q_{d}$ and $Q_{c}$, respectively, PDMS microdroplets with four sizes were obtained simultaneously, as shown in Fig. $6 A, B, C$ and $D$, respectively.

The smallest and largest droplets were formed in the 34-30G and 23G-20G channels, respectively. Table 1 lists the measured droplet sizes and their CVs calculated by Eq. (1). As shown from Fig. 6 and Table 1, at the same flow rates, larger needles are able to generate droplets with a larger diameter. For the same needles, droplet sizes are controlled by the flow rates. For example, in the case of 23G-20G, the droplet sizes grew from $399 \mu \mathrm{m}$ to $438 \mu \mathrm{m}$ when we increased $Q_{d}$ from 20 to $40 \mu \mathrm{L} / \mathrm{min}$ while keeping $\mathrm{Q}_{c}$ unchanged. CVs of all groups were less than $3 \%$, indicating that highly monodispersed droplets were formed. 


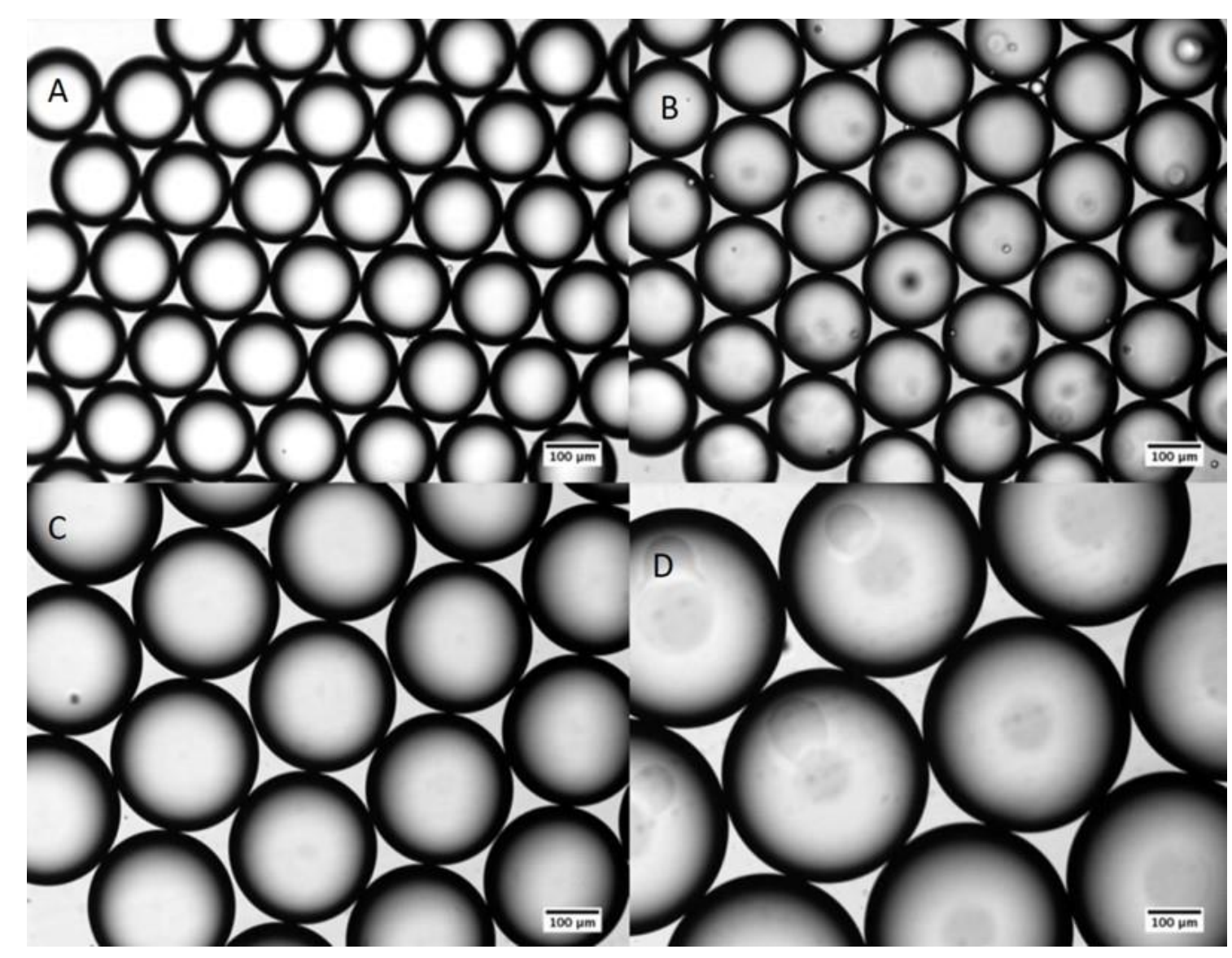

Figure 6. Using TWC for a parallel connection of four-needle devices to produce various sizes of PDMS single emulsion microdroplets simultaneously. The optical images of PDMS microdroplets fabricated by 34G-30G, 32G-27G, 25G-21G and 23G-20G are shown in (A), (B), (C) and (D), respectively, with $Q_{d}=20$ $\mu \mathrm{L} / \mathrm{min}$ and $\mathrm{Q}_{\mathrm{c}}=200 \mu \mathrm{L} / \mathrm{min}$.

Table 1. Diameters and CV values of the PDMS microdroplets generated at different flow rates by the four-needle devices, i.e., 34G-30G, 32G-27G, 25G-21G and 23G-20G, which were connected in a parallel way.

\begin{tabular}{llll} 
Needles & $\mathrm{Q}(\mu \mathrm{L} / \mathrm{min})$ & $\mathrm{D}(\mu \mathrm{m})$ & $\mathrm{CV}(\%)$ \\
\hline 23G-20G & $20-200$ & 398.9 & 1.4 \\
& $30-200$ & 427.4 & 2.9 \\
& $40-200$ & 438.4 & 2.2 \\
\hline 25G-21G & $20-200$ & 268.5 & 0.5 \\
& $30-200$ & 273.4 & 0.3 \\
& $40-200$ & 279.4 & 0.6 \\
\hline 32G-27G & $20-200$ & 185.6 & 0.5 \\
& $30-200$ & 186.1 & 0.7
\end{tabular}




\begin{tabular}{llll} 
& $40-200$ & 193.3 & 0.6 \\
\hline 34G-30G & $20-200$ & 174.5 & 0.7 \\
& $30-200$ & 175.4 & 0.7 \\
$40-200$ & 177.0 & 0.6 \\
\hline
\end{tabular}

Table 2. Generation rates and size of droplets produced by TWC, FWC and SWMC.

\begin{tabular}{lccc}
\hline Parallel Connections & Production Rates (drops/min) & $\mathrm{D}(\mu \mathrm{m})$ & $\mathrm{CV}(\%)$ \\
\hline TWC (4 channels) & 660 & 653.6 & 1.3 \\
FWC (8 channels) & 1370 & 657.1 & 2.4 \\
SWMC ( 2 channels) & 310 & 650 & 2.8 \\
\hline
\end{tabular}

\subsection{Single cored double emulsion formation}

The 32G-21G-20G and 30G-21G-20G needle devices were utilized to generate W/O/W double emulsions, with flow rate combination of 8,20 and $200 \mu \mathrm{L} / \mathrm{min}$ for $\mathrm{Q}_{\mathrm{i}}, \mathrm{Q}_{\mathrm{m}}$ and $\mathrm{Q}_{0}$, respectively. Red and black dyes were mixed with DI water as the inner phase and employed for the upper and lower droplet makers, respectively, to distinguish and identify the cores. In order to improve the hydrophobicity, the middle needles were immersed in OTS solutions for 30 minutes in room temperature following a heat treatment of $80{ }^{\circ} \mathrm{C}(30 \mathrm{~min})$ to allow the evaporation of the residue solution. Due to the intrinsic hydrophilic properties of stainless-steel part of the needles, the surface wettability modification of inner and outer needle can be neglected for this W/O/W template. The middle phase was PDMS with a 10:2 wt\% for base and curing agent, while DI water with PVA (5 wt\%) was chosen as the outer phase. The droplets formed in those two channels were captured in Fig. 7A and B. Since the flow conditions were identical for both emulsifiers, the only difference was the inner needle diameter-30G is slightly larger than 32G. As speculated, the larger inner channel was beneficial for the inner phase to grow and break up with larger cores, as shown in Fig. 7A and B and Table 3. Additionally, the obtained CVs of overall and core sizes suggest the droplets generated by two modified needle devices connected in a shunt way have a narrow size distribution. 


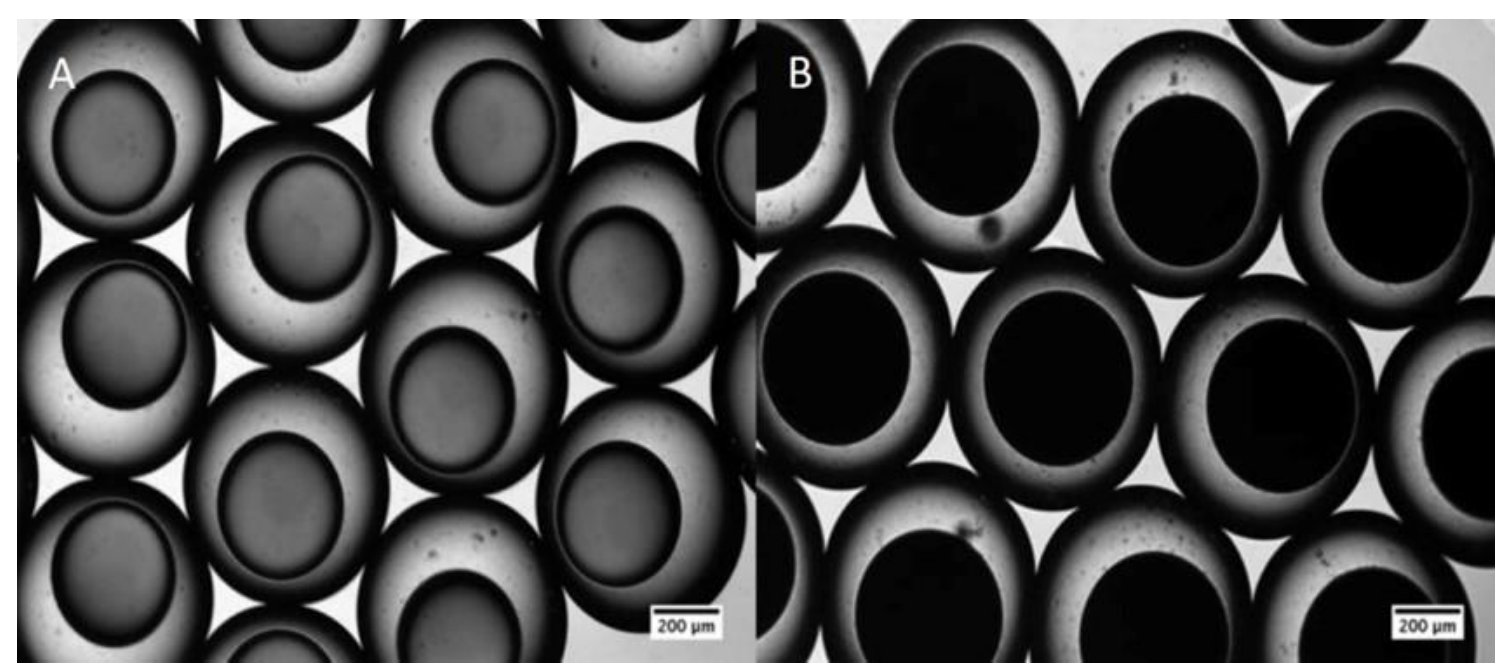

Figure 7. Optical images of the fabricated single cored double emulsion microdroplets (A) $32 \mathrm{G}-21 \mathrm{G}-20 \mathrm{G}$ and (B) 30G-21G-20G, using three-way link for two channels in parallel.

Table 3. Measured sizes and CVs of double emulsion PDMS microdroplets formed by parallelly connected needle devices.

\begin{tabular}{ccccc} 
Needles & $\mathrm{D}_{\mathrm{o}}(\mu \mathrm{m})$ & $\mathrm{CV}(\%)$ & $\mathrm{D}_{\mathrm{c}}(\mu \mathrm{m})$ & $\mathrm{CV}(\%)$ \\
\hline 30G-21G-20G & 690.3 & 3.0 & 466.5 & 3.2 \\
32G-21G-20G & 642.2 & 1.6 & 391.4 & 1.0 \\
\hline
\end{tabular}

\subsection{Multiple cored double emulsion formation}

The versatile needle device is competent to generate multiple cored double emulsions. To examine its effeteness for the production of droplets, we used two kinds of PDMS with different viscosities by preparing mixture of PDMS and curing agent at weight ratios of 10:1 and 10:2, respectively, labelled as $\mathrm{PDMS}_{1}$ and $\mathrm{PDMS}_{2}$, in the following discussion. Two identical needle devices made of 34-21G-20G needles connected in a shunt way were used in the experiments for producing multiple cored double emulsions.

$P D M S_{1}$ and $P D M S_{2}$ were pumped into two sets of droplet generators defined as $D G_{1}$ and $D G_{2}$, respectively. To enhance the contrast and facilitate visualization when the droplets are formed, red and black inks were mixed with $\mathrm{DI}$ water as the inner phases in $D G_{1}$ and $D G_{2}$, respectively. The DI water with 5 wt \% PVA surfactant was used as the outer phase. Double emulsions with four cores can be formed using $D_{1} G_{1}$ while single core was formed using $\mathrm{DG}_{2}$ as shown in Fig. $8 \mathrm{~A}$ and $\mathrm{B}$, respectively, at the same flow conditions when $Q_{i}=8 \mu \mathrm{L} / \mathrm{min}, \mathrm{Q}_{\mathrm{m}}=20 \mu \mathrm{L} / \mathrm{min}$ and $\mathrm{Q}_{0}=200 \mu \mathrm{L} / \mathrm{min}$. The encapsulation of inner cores at higher flow rate of middle phase is shown in Fig. $8 \mathrm{C}$ and $\mathrm{D}$ when $\mathrm{Q}_{\mathrm{m}}=25 \mu \mathrm{L} / \mathrm{min}$ and in Fig. $8 \mathrm{E}$ and $\mathrm{F}$ when $\mathrm{Q}_{\mathrm{m}}=30$ $\mu \mathrm{L} / \mathrm{min}$. The breakup of liquid thread is driven by the balance among interfacial tension, inertia and viscous shear stress. Decreasing the interfacial tension would slow down the breakup process [48-50], thus reducing the possibility for formed droplets to be encapsulated by the middle phase. We attribute the experimentally observed difference in number of encapsulated cores in $D G_{1}$ and $D G_{2}$ at the same flow 
conditions to the difference in interfacial tension. To verify the hypothesis, we measured the Capillary number of middle phase defined by

$$
C a_{m}=\frac{\mu_{m} \times V_{m}}{\sigma_{m o}}
$$

where $\mu, \mathrm{V}$ and $\sigma$ are the viscosity, velocity and interfacial tension, respectively. The subscript $\mathrm{m}$ and o denote the middle and outer phase, respectively. In our experiments, the viscosities of PDMS ${ }_{1}$ and PDMS 2 were 3.76 and $2.01 \mathrm{~Pa} \mathrm{~s}$, and their interfacial tensions with water were 15.2 and $14.01 \mathrm{mN} / \mathrm{m}$, respectively. Capillary number of the inner and outer phases remained constant except for that of the middle phase through our experiments. For convenience, we use $\mathrm{Ca}_{\mathrm{m} 1}$ and $\mathrm{Ca}_{2}$ to denote the Capillary numbers of the middle phase in $D G_{1}$ and $D G_{2}$, respectively. $\mathrm{Ca}_{\mathrm{m} 1}$ was $2.91 \times 10^{-4}$ and $\mathrm{Ca}_{2}$ was $1.69 \times 10^{-4}$ when $\mathrm{Q}_{\mathrm{i}}=8 \mu \mathrm{L} / \mathrm{min}$, $Q_{m}=20 \mu \mathrm{L} / \mathrm{min}$ and $Q_{0}=200 \mu \mathrm{L} / \mathrm{min}$. The number of encapsulated inner cores $(\mathrm{N})$ in double emulsion versus different Capillary number of the middle phase is shown in Fig.9, and the measured droplet sizes as well as size distribution is listed in Table 4. More inner cores were encapsulated in double emulsion droplets when $Q_{m}$ increased while $Q_{i}$ and $Q_{o}$ were kept constant. When $Q_{m}$ is increased, corresponding to a larger $\mathrm{Ca}_{\mathrm{m}}$, it will facilitate more cores to be captured in a droplet, as verified by Fig. $8 \mathrm{C}$ and $\mathrm{D}$, where five cores were encapsulated in $D_{1}\left(C_{m 1}=3.63 \times 10^{-4}\right)$ and two cores for $D_{2}\left(C_{m 2}=2.11 \times 10^{-4}\right)$ when $Q_{m}$ increased up to $25 \mu \mathrm{L} / \mathrm{min}$. When $\mathrm{Q}_{\mathrm{m}}$ increased to $30 \mu \mathrm{L} / \mathrm{min}$, the number of cores in a droplet became six for $D G_{1}$ and three for $D_{2}$, see Fig. $8 E$ and $F$. A larger $C_{m}$ implies that a higher shear force is exerted to the inner fluid, resulting in smaller sizes of inner droplets. This is consistent with our experimental observations shown in Fig. 8 as well as the measured data in Table 4.

However, increase of $Q_{m}$ is capped by instability of droplets production. In our experiments, when $Q_{m}$ in $D_{1}$ reached $35 \mu \mathrm{L} / \mathrm{min}$, stabilization of double emulsion with controllable number of cores could not be guaranteed as seven or eight cores were found in the same batch as shown in Fig. S4. The value of $\mathrm{Cam}_{\mathrm{m}}$ in this case was $5.09 \times 10^{-4}$, larger than the suggested value, i.e., $\mathrm{Ca}=4.8 \times 10^{-4}$ for monodispersed double emulsion droplets with stable number of inner cores [50]. For $\mathrm{DG}_{2}$, since the viscosity of middle phase is lower than that in $D G_{1}, Q_{m}$ can increase until it is equal to $55 \mu \mathrm{L} / \mathrm{min}$ with six cores encapsulated. It occurred for formation of double emulsions with unstable number of cores when $Q_{m}$ was beyond 55 $\mu \mathrm{L} / \mathrm{min}$. This result also agreed well with that reported by Nabavi et al [50], where unstable number of cores were encapsulated when $\mathrm{Q}_{m}=57 \mu \mathrm{L} / \mathrm{min}$, and $\mathrm{Ca}=4.81 \times 10^{-4}$. Interestingly, decreasing $\mathrm{Q}_{\mathrm{m}}$ to 10 $\mu \mathrm{L} / \mathrm{min}$ in $D_{1}$ resulted in the core number equal to two, but the core number could not be well adjusted when further reducing $Q_{m}$ below $9 \mu \mathrm{L} / \mathrm{min}\left(\mathrm{Ca}_{\mathrm{m} 1}=1.31 \times 10^{-4}\right)$. As to $\mathrm{DG}_{2}, \mathrm{Q}_{\mathrm{m}}$ should be set beyond 17 $\mu \mathrm{L} / \mathrm{min}$ (corresponding to $\mathrm{Ca}_{\mathrm{m} 2}=1.44 \times 10^{-4}$ ) to maintain formation with stable number of cores. In summary, the maximum number of stable inner cores from the developed device was six. Further increase in $Q_{m}$ led to encapsulation of more inner cores yet with high polydispersity for the size of inner cores and outer droplets. Therefore, the viable $\mathrm{Ca}_{\mathrm{m}}$ should range from $1.31 \times 10^{-4}$ to $4.64 \times 10^{-4}$ to obtain stable and controllable numbers of cores for the needle device as shown in Fig. 9. More importantly, the measured sizes in Table 4 demonstrate that monodispersed double emulsion droplets with stable number of cores can be generated in the parallelization mode. Multiple such sets of double emulsion generators can run in parallel to produce double emulsions with various number of cores simultaneously via controlling the Capillary number of the middle phase. Additionally, the PDMS microdroplets could be solidified into 
microparticles under thermal conditions such as under $70^{\circ} \mathrm{C}$ for 2 hours. Cross section view of a solidified five-cored PDMS double emulsion templated microparticle taken by SEM was shown as an example in Fig. S5.

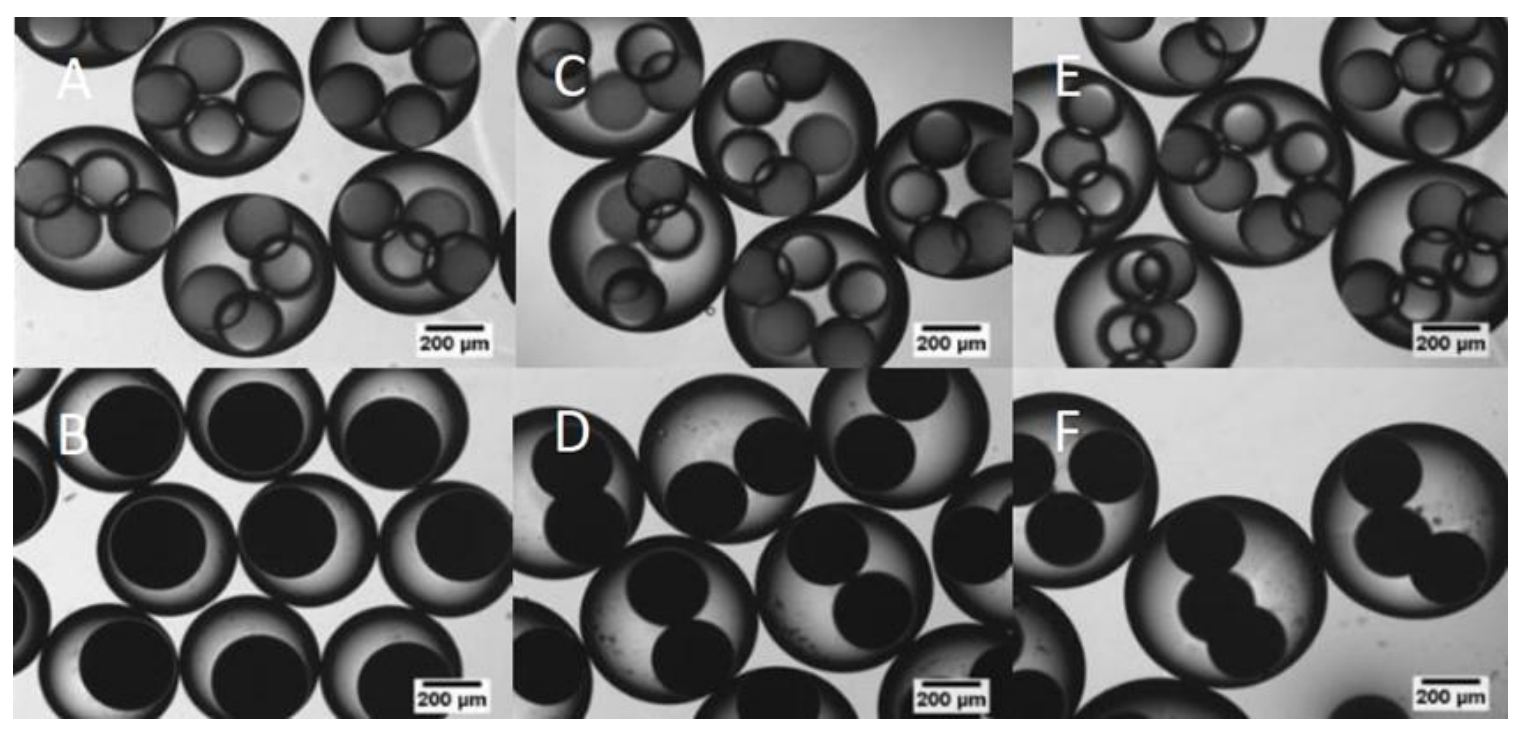

Figure 8. Optical images of the formed double emulsions with different number of cores by tuning $Q_{m}$ while fixing $Q_{i}=8 \mu \mathrm{L} / \mathrm{min}$ and $Q_{0}=200 \mu \mathrm{L} / \mathrm{min}$. (A) and (B) The flow rate is $20 \mu \mathrm{L} / \mathrm{min}$ for $Q_{m}$; (C) and (D) The flow rate is $25 \mu \mathrm{L} / \mathrm{min}$ for $Q_{m}$; $(E)$ and $(F)$ The flow rate is $30 \mu \mathrm{L} / \mathrm{min}$ for $Q_{m}$. The inner cores were dyed by inks to increase contrast of images. $D G_{1}$ was used for (A), (C) and (E); and $D G_{2}$ was used for (B), (D) and (F). All sub-figures share the same scale bar denoting 200 microns.

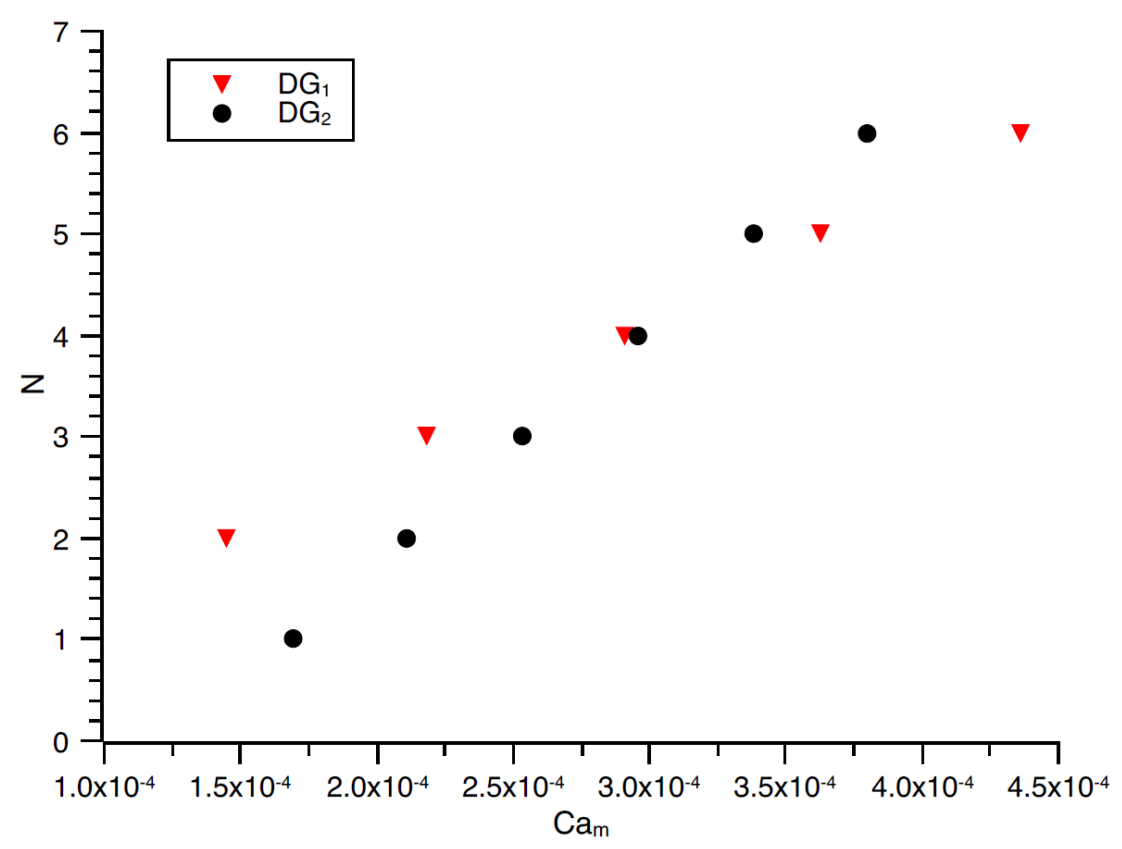

Figure 9. Controllable number of inner cores against Capillary number of middle phase.

Table 4. Measured sizes and CV values of multiple-cored double emulsion PDMS microdroplet produced using parallelly connected needle devices. 


\begin{tabular}{lcccccc} 
& $\mathrm{Q}(\mu \mathrm{l} / \mathrm{min})$ & Cores & $\begin{array}{c}\mathrm{D}_{0} \\
(\mu \mathrm{m})\end{array}$ & $\begin{array}{c}\mathrm{CV} \\
(\%)\end{array}$ & $\begin{array}{c}\mathrm{D}_{\mathrm{c}} \\
(\mu \mathrm{m})\end{array}$ & $\begin{array}{c}\mathrm{CV} \\
(\%)\end{array}$ \\
\hline $\mathrm{DG}_{1}$ & $8-20-200$ & 4 & 594.3 & 1.0 & 239.6 & 1.9 \\
$\mathrm{DG}_{2}$ & $8-20-200$ & 1 & 492.8 & 0.8 & 338.9 & 1.1 \\
\hline $\mathrm{DG}_{1}$ & $8-25-200$ & 5 & 635.1 & 1.1 & 230.3 & 2.5 \\
$\mathrm{DG}_{2}$ & $8-25-200$ & 2 & 613.7 & 1.1 & 281.9 & 1.9 \\
\hline $\mathrm{DG}_{1}$ & $8-30-200$ & 6 & 686.1 & 1.6 & 223.7 & 2.7 \\
$\mathrm{DG}_{2}$ & $8-30-200$ & 3 & 659.9 & 2.5 & 274.3 & 2.6 \\
\hline
\end{tabular}

\section{Conclusions}

We reported in this paper a facile way to form highly monodispersed multiple cored emulsions with $\mathrm{CV}<3 \%$ using needle-based microfluidic devices connected in a parallel mode. Through use of the shunted TWC and the 3D printed FWC, the droplet production rates were improved almost fourfold (over 660 droplets/min) and eightfold (over 1300 droplets/min) in comparison to that of a single device (160 droplets/min) respectively, while the monodispersity was not compromised, attributing to the precise and independent control of flow conditions. Four groups of PDMS microdroplets with distinct sizes were obtained simultaneously using TWCs. Our microfluidic system leads to formation of groups of droplets with various sizes yet each group has high monodispersity, thereby enabling concurrent batch bioassays for pharmaceutical drug development. In addition, an off-the-shelf six-way microfluidic component was proposed to integrate two droplet makers. Parallelization of double emulsion devices was realized by connecting two set of devices with different needle sizes, leading to formation of multi-cored double emulsions, the number of encapsulated inner cores can be controlled by tuning the Capillary number of middle phase in a range from $1.31 \times 10^{-4}$ to $4.64 \times 10^{-4}$ via adjusting the flow rates of the middle phase. Both the core and overall droplet sizes were found monodispersed. This allows for development of drug carriers with flexibility in control over the dosage and drug loadings. This is of crucial importance for drug screening or drug-dosing tests. Our approach is robust and highly feasible for mass production. Our work will inspire the development of biocompatible materials with highly complex structures.

\section{Conflicts of interest}

The authors declare no conflict of interest.

\section{Acknowledgements}

This research was supported by Zhejiang Provincial Natural Science Foundation of China under Grant No. LY19E060001 and LQ19F050003, Ningbo Science and Technology Bureau under Service Industry Science \&Technology Programme with project code 2019F1030, Ningbo Science and Technology Bureau Technology Innovation Team under Grant No. 2017C510001, Shenzhen Natural Science 
Foundation under Grant No. JCYJ201908083000825, Research Seed and Supplementary Support Fund from Nottingham China Health Institute, as well as Zhejiang Provincial First-Class Discipline Construction Grant: Materials Science and Engineering, University of Nottingham Ningbo China. Z. Lian acknowledges the financial support from the International Doctoral Innovation Centre, Ningbo Education Bureau, Ningbo Science and Technology Bureau, and the University of Nottingham. The authors acknowledge the contribution by Ms. Xinyuan Wang in this research project.

\section{References}

[1] Teh, S. Y., Lin, R., Hung, L. H., Lee, A. P., Lab Chip 2008, 8, 198-220.

[2] Whitesides, G. M., Nature 2006, 442, 368-373.

[3] Huebner, A., Sharma, S., Srisa-Art, M., Hollfelder, F., Edel, J. B., deMello, A. J., Lab on a Chip 2008, 8, 1244-1254.

[4] Wang, W., Zhang, M. J., Chu, L. Y., Curr Opin Pharmacol 2014, 18, 35-41.

[5] Zhao, C.-X., Middelberg, A. P. J., Chemical Engineering Science 2011, 66, 1394-1411.

[6] Utada, A. S., Chu, L. Y., Fernandez-Nieves, A., Link, D. R., Holtze, C., Weitz, D. A., MRS Bulletin 2007, 32, 702-708.

[7] Kim, S.-H., Kim, J. W., Cho, J.-C., Weitz, D. A., Lab on a Chip 2011, 11, 3162-3166.

[8] He, T., Liang, Q., Zhang, K., Mu, X., Luo, T., Wang, Y., Luo, G., Microfluidics and Nanofluidics 2011, 10, 1289-1298.

[9] Kastner, E., Kaur, R., Lowry, D., Moghaddam, B., Wilkinson, A., Perrie, Y., Int J Pharm 2014, 477, 361368.

[10] Song, H., Chen, D. L., Ismagilov, R. F., Angewandte Chemie International Edition 2006, 45, 7336-7356.

[11] Ward, T., Faivre, M., Stone, H. A., Langmuir 2010, 26, 9233-9239.

[12] Holtze, C., Journal of Physics D: Applied Physics 2013, 46, 114008.

[13] Kong, T., Wu, J., To, M., Wai Kwok Yeung, K., Cheung Shum, H., Wang, L., Biomicrofluidics 2012, 6, 034104.

[14] Vladisavljević, G., Nuumani, R., Nabavi, S. A., Micromachines 2017, 8, 75.

[15] Nisisako, T., Ando, T., Hatsuzawa, T., Lab Chip 2012, 12, 3426-3435.

[16] Ofner, A., Mattich, I., Hagander, M., Dutto, A., Seybold, H., Rühs, P. A., Studart, A. R., Advanced Functional Materials 2019, 29, 1806821.

[17] Ofner, A., Moore, D. G., Rühs, P. A., Schwendimann, P., Eggersdorfer, M., Amstad, E., Weitz, D. A., Studart, A. R., Macromolecular Chemistry and Physics 2017, 218, 1600472.

[18] Kim, S.-H., Kim, J. W., Kim, D.-H., Han, S.-H., Weitz, D. A., Microfluidics and Nanofluidics 2013, 14, 509514.

[19] Liu, D., Cito, S., Zhang, Y., Wang, C.-F., Sikanen, T. M., Santos, H. A., Advanced Materials 2015, 27, 2298-2304.

[20] Liu, D., Wang, L., Zhong, R., Li, B., Ye, N., Liu, X., Lin, B., J Biotechnol 2007, 131, 286-292.

[21] Tottori, N., Nisisako, T., Sensors and Actuators B: Chemical 2018, 260, 918-926.

[22] Conchouso, D., Castro, D., Khan, S. A., Foulds, I. G., Lab Chip 2014, 14, 3011-3020.

[23] Han, T., Zhang, L., Xu, H., Xuan, J., Chemical Engineering Journal 2017, 326, 765-773.

[24] Zhou, P., Tarlet, D., Wei, M., Fan, Y., Luo, L., Chemical Engineering Research and Design 2017, 121, 233-244.

[25] Arriaga, L. R., Amstad, E., Weitz, D. A., Lab Chip 2015, 15, 3335-3340.

[26] Arriaga, L. R., Datta, S. S., Kim, S. H., Amstad, E., Kodger, T. E., Monroy, F., Weitz, D. A., Small 2014, 10, 950-956.

[27] Bardin, D., Kendall, M. R., Dayton, P. A., Lee, A. P., Biomicrofluidics 2013, 7, 34112. 
[28] Cohen, C., Giles, R., Sergeyeva, V., Mittal, N., Tabeling, P., Zerrouki, D., Baudry, J., Bibette, J., Bremond, N., Microfluidics and Nanofluidics 2014, 17, 959-966.

[29] Lim, J. M., Bertrand, N., Valencia, P. M., Rhee, M., Langer, R., Jon, S., Farokhzad, O. C., Karnik, R., Nanomedicine 2014, 10, 401-409.

[30] Romanowsky, M. B., Abate, A. R., Rotem, A., Holtze, C., Weitz, D. A., Lab Chip 2012, 12, 802-807.

[31] Amstad, E., Chemama, M., Eggersdorfer, M., Arriaga, L. R., Brenner, M. P., Weitz, D. A., Lab on a Chip 2016, 16, 4163-4172.

[32] Headen, D. M., García, J. R., García, A. J., Microsystems \& Nanoengineering 2018, 4, 17076.

[33] Vian, A., Reuse, B., Amstad, E., Lab on a Chip 2018, 18, 1936-1942.

[34] Akbari, S., Pirbodaghi, T., Kamm, R. D., Hammond, P. T., Lab Chip 2017, 17, 2067-2075.

[35] Amstad, E., Chen, X., Eggersdorfer, M., Cohen, N., Kodger, T. E., Ren, C. L., Weitz, D. A., Phys Rev E 2017, 95, 043105.

[36] Baudoin, R., Alberto, G., Paullier, P., Legallais, C., Leclerc, E., Sensors and Actuators B: Chemical 2012, 173, 919-926.

[37] Yadavali, S., Jeong, H. H., Lee, D., Issadore, D., Nat Commun 2018, 9, 1222.

[38] Bodas, D., Khan-Malek, C., Sensors and Actuators B: Chemical 2007, 123, 368-373.

[39] Anna, S. L., Bontoux, N., Stone, H. A., Applied Physics Letters 2003, 82, 364-366.

[40] Lee, J. N., Park, C., Whitesides, G. M., Analytical Chemistry 2003, 75, 6544-6554.

[41] Yoo, S. H., Cohen, C., Hui, C.-Y., Polymer 2006, 47, 6226-6235.

[42] Koh, K.-S., Chin, J., Chia, J., Chiang, C.-L., Micromachines 2012, 3, 427-441.

[43] Li, T., Zhao, L., Liu, W., Xu, J., Wang, J., Lab on a Chip 2016, 16, 4718-4724.

[44] Lian, Z., Ren, Y., He, J., Chen, G. Z., Koh, K. S., Microfluidics and Nanofluidics 2018, 22, 145.

[45] Tran, D. N. H., Kabiri, S., Sim, T. R., Losic, D., Environmental Science: Water Research \& Technology 2015, 1, 298-305.

[46] Choi, S.-J., Kwon, T.-H., Im, H., Moon, D.-I., Baek, D. J., Seol, M.-L., Duarte, J. P., Choi, Y.-K., ACS Applied Materials \& Interfaces 2011, 3, 4552-4556.

[47] Park, I., Efimenko, K., Sjöblom, J., Genzer, J., Journal of Dispersion Science and Technology 2009, 30, 318-327.

[48] Chu, L.-Y., Utada, A. S., Shah, R. K., Kim, J.-W., Weitz, D. A., Angewandte Chemie International Edition 2007, 46, 8970-8974.

[49] Kim, S.-H., Hwang, H., Lim, C. H., Shim, J. W., Yang, S.-M., Advanced Functional Materials 2011, 21, 1608-1615.

[50] Nabavi, S. A., Vladisavljević, G. T., Manović, V., Chemical Engineering Journal 2017, 322, 140-148.

\section{List of Abbreviations}

\begin{tabular}{ll}
\hline AD & Average Diameter, $\mu \mathrm{m}$ \\
$\mathrm{Ca}$ & Capillary number, - \\
$\mathrm{Cam}$ & Capillary number of middle phase, - \\
$\mathrm{CP}$ & Continuous Phase, - \\
$\mathrm{CV}$ & Coefficient of Variation, $\%$ \\
$\mathrm{D}$ & Diameter, $\mu \mathrm{m}$ \\
$\mathrm{DI}$ & Deionized water, -
\end{tabular}


DP Dispersed Phase, -

FWC Four-way Connector, -

I. D. Inner Diameter, $\mu \mathrm{m}$

N Number of inner cores, -

O. D. Outer Diameter, $\mu \mathrm{m}$

OTS Octadecyltrichlorosilane

PDMS Polydimethylsiloxane, -

PMMA Polymethyl methacrylate, -

PP Polypropylene, -

PVA Polyvinyl alcohol, -

Q Flow rate, $\mu \mathrm{L} / \mathrm{min}$

SLA Stereo Lithography Apparatus, -

STDV Standard Deviation, -

SWMC Six-Way Microfluidic Component

TWC Two-way Connector, -

W/O Water-in-Oil, -

W/O/W Water-in-Oil-in-Water, -

Subscripts

c core

i inner phase

$\mathrm{m} \quad$ middle phase

o outer phase/overall droplet 